\title{
Study on the Effectiveness Evaluation Method Based on the Combination of AHP and Grey Interval Correlation
}

\author{
Xu Shi-hong ${ }^{1}$, Zhao Wei-bin ${ }^{2}$, Huang Guo-qing ${ }^{1,2}$ \\ ${ }^{1}$ Department of Aeronautic Electronic Engineering, the First Aeronautical College of Air Force, Xinyang, China \\ ${ }^{2}$ School of Information Engineering, Zhengzhou University, Zhengzhou, China
}

\section{Email address:}

zzuweibin@163.com (Zhao Wei-bin),hguoqing@163.com (Huang Guo-qing)

To cite this article:

Xu Shi-hong, Zhao Wei-bin, Huang Guo-qing. Study on the Effectiveness Evaluation Method Based on the Combination of AHP and Grey Interval Correlation. Journal of Electrical and Electronic Engineering. Vol. 3, No. 5, 2015, pp. 177-180. doi: 10.11648/j.jeee.20150305.23

\begin{abstract}
Aiming at the problem of using single judging value for the expert score of the traditional effectiveness evaluation, the expert's score data are not reliable; an improved effectiveness evaluation method based on the combination of AHP and grey interval correlation analysis is proposed. Interval number is used for the expert score in the improved method, the influence of the subjective fuzziness and objective fuzziness on the expert score using the single judging value is overcame. Interval number improved the reliability of the evaluation data. Combination weighting approach considering the influence of subjective and objective factors, the weight is more reasonable. The concrete steps of the improved effectiveness evaluation method are given. The improved method is proved to be more reasonable and reliable through an example.
\end{abstract}

Keywords: AHP, Interval Number, Gray Relational Degree, Combination Weighting Approach, Effectiveness Evaluation

\section{Introduction}

The gray system theory proposed by Professor Deng Ju-long is a method of studying on the uncertain problem whose part of the information is known, while some of the information is unknown, "small sample" and " Poor information" [1]. Grey relational degree is often used to indicate the degree of correlation or the degree of similarity between grey system factors, and the gray relational degree plays an important role in the quantitative analysis of grey system theory. The results of the grey relational degree efficiency evaluation are based on the expert's scoring value, so the accuracy of the expert's scoring value directly affects the reliability of the results.

At present, Chen Kai, Zhao Yu-hui, Du Jun proposed that the index was given different weights according to the actual important degree of the different index, to overcome the problem of using same weight and poor objectivity of the index in the calculation of correlation degree [2]. Chang Shuang-jun and Ma Jin-ya used synthetical optimization based on gray associated method for effectiveness evaluation of weapon system to avoid the results of effectiveness evaluation which is too partial or inferior to make the evaluation results more reasonable [3]. Ning Xiao-lei et al. proposed simulation models based on the improved grey relational analysis, which combines the geometric similarity and numerical similarity, and reduces the risk of conventional grey correlation analysis, and improves the credibility of the results of the verification [4]. ChihHsuan Wang used the grey correlation analysis method to optimize the green production project, which can use the minimum energy consumption to get lean production [5] and so on.

In the above researches the experts' score data used a single value, however, it does not meet the people's fuzzy thinking habits [6]. Because of the ambiguity of the human mind and the influence of the objective factors, the value of the index judged by many people is a certain interval [7], and the interval number is more able to reflect the reliability of expert scoring. Therefore, in order to make the evaluation result more reasonable and credible, this paper proposes a method of combining AHP with grey interval correlation.

\section{Analysis on the Improved Method of Effectiveness Evaluation}

The gray system is complex and information incomplete, it has a large number of factors, complex logic relationship. Therefore, it is very difficult to evaluate the effectiveness of the gray system. The analytic hierarchy process (AHP) method decomposes the complex system into several elements, such as 
target, criterion, index, etc., and then, according to the different attributes, these elements are arranged into a hierarchical relationship. It is the core idea of the AHP to establish an effective and reasonable hierarchical relationship.

In the process of the effectiveness evaluation, the index weight is very important, and it has a great influence on the evaluation results. Therefore, the determination of the weights of the index should be scientific and reasonable, and the reliability of the results for the effectiveness evaluation is related to it. At present, there are some subjective weighting methods, such as analytic hierarchy process, expert investigation method and so on. Objective weighting method includes the method of maximizing deviation, information entropy method, etc.. In order to make the results more reasonable and reliable, combination weighting approach can be used to not only consider the preference of the experts to the index, but also reduce the subjective randomness.

In the improved effectiveness evaluation method based on the combination of AHP and grey interval correlation analysis, reasonable hierarchical relationship is established by AHP. The expert points are unified by using the evaluation interval number so the accuracy of the evaluation data are promoted. By using the combination weight approach to determine the weight, and by taking into account the subjective and objective factors, the weight is more reasonable. Extraction of the information vector of interval numbers after weighted [8], the vector similarity algorithm has represented the gray relational degree of the comparison scheme's information vector and the reference scheme's information vector (the ideal optimal or worst scheme).

\section{Concrete Steps of the Improved Effectiveness Evaluation Method}

According to the above analysis, the concrete steps of the effectiveness evaluation method of the combination of AHP and grey interval correlation analysis are as follows:

Step 1: use AHP method to establish the hierarchical relationship

According to the principle of AHP method, a reasonable hierarchical relationship is set up, which includes the target layer, the criterion layer, and the index layer. The object to be evaluated is recorded as $A=\{A 1, A 2, A 3, \ldots, A n\}$; the criteria layer index is recorded as $\mathrm{B}=\{\mathrm{B} 1, \mathrm{~B} 2, \ldots \mathrm{Bf}\}$; the index layer is denoted as $\mathrm{C}=\{\mathrm{C} 1, \mathrm{C} 2, \ldots \mathrm{Cm}\}$. The corresponding index interval number of the underlying index $\mathrm{c}_{\mathrm{j}}$ in the scheme $A_{\mathrm{i}}$ is recorded as $\mathrm{a}_{\mathrm{ij}}=\left(\underline{\mathrm{a}_{\mathrm{ij}}}, \overline{\mathrm{a}_{\mathrm{ij}}}\right)$ where $\underline{\mathrm{a}_{\mathrm{ij}}}$ and $\overline{\mathrm{a}_{\mathrm{ij}}}$ expressed the lower and upper bounds of the interval respectively.

Step 2: obtain expert evaluation interval number matrix and normalized processing

The evaluation interval number of the underlying index $\mathrm{c}_{\mathrm{j}}$ of the expert $\mathrm{K}$ treatment evaluation scheme $A_{\mathrm{i}}$ is recorded

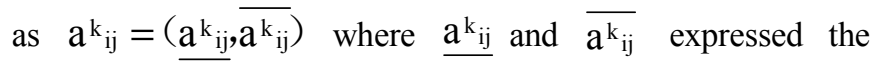

lower and upper bounds of the interval respectively.

$$
\begin{gathered}
a_{i j}=\frac{\sum_{m=1}^{k} \frac{a^{m_{i j}}}{k}}{k} \\
\overline{a_{i j}}=\frac{\sum_{m=1}^{k} \overline{a^{m_{i j}}}}{k}
\end{gathered}
$$

the formula (1)and(2), $\mathrm{K}$ is the total number of experts involved in the assessment.

The corresponding index interval number of the underlying index $\mathrm{c}_{\mathrm{j}}$ in the scheme $A_{\mathrm{i}}$ is recorded as $a_{i j}=\left(\underline{a_{i j}}, \overline{a_{i j}}\right)$ where $\underline{a_{i j}}$ and $\overline{a_{i j}}$ expressed the lower and upper bounds of the interval respectively. So the experts evaluate the interval number matrix:

$$
X=\left[\begin{array}{llll}
a_{11} & a_{12} & \cdots & a_{1 j} \\
a_{21} & a_{22} & \cdots & a_{2 j} \\
\vdots & \vdots & \cdots & \vdots \\
a_{n 1} & a_{n 2} & \cdots & a_{n j}
\end{array}\right]
$$

Because the physical meaning of each factor is different, its mathematical dimension is not necessarily the same, thus the number of data size is different, therefore it is difficult to get the correct comparative results in comparison. So the dimension of the data in the matrix is eliminated and it is transformed into a comparable data sequence. For the benefit and the cost type index, the formula (4) and the formula (5) are used to normalize the data in order to make each index value in the range of $[0,1]$.

$$
\begin{aligned}
& b_{i j}=\frac{a_{i j}}{\max _{i=1}^{n}\left(\overline{a_{i j}}\right)} \\
& b_{i j}=\frac{\min _{i=1}^{n}\left(\underline{a_{i j}}\right)}{a_{i j}}
\end{aligned}
$$

Get the score matrix of the expert after normalize

$$
Y=\left[\begin{array}{llll}
\mathrm{b}_{11} & \mathrm{~b}_{12} & \cdots & \mathrm{b}_{1 \mathrm{j}} \\
\mathrm{b}_{21} & \mathrm{~b}_{22} & \cdots & \mathrm{b}_{2 \mathrm{j}} \\
\vdots & \vdots & \cdots & \vdots \\
\mathrm{b}_{\mathrm{n} 1} & \mathrm{~b}_{\mathrm{n} 2} & \cdots & \mathrm{b}_{\mathrm{nj}}
\end{array}\right]
$$

Where $b_{i j}=\left(b_{i j}, \overline{b_{i j}}\right)$ 。

Step 3: determine the weights of the index

(1) Determine the subjective weight

In this paper, the subjective weight is determined by analytic hierarchy process. On the same level, the importance of each factor is relavent to the upper level of the importance 
of the two comparison, getting the judgment matrix, the use of square method to calculate the eigenvalues and eigenvectors of the matrix, and the weight of the judgment matrix consistency test. When the consistency check probability $\mathrm{CR}<0.1$, then the judgment matrix meets the consistency, get the subjective weight $\mathrm{w}_{\mathrm{s}}=\left(\begin{array}{llll}\mathrm{w}_{\mathrm{s} 1} & \mathrm{w}_{\mathrm{s} 2} \mathrm{~L} & \mathrm{w}_{\mathrm{sm}}\end{array}\right)$

(2) Determine the objective weight

In this paper, the objective weight is determined by entropy method. The information entropy and the weights of the index are calculated by the formula (7) and (8) respectively.

$$
\begin{gathered}
E_{\mathrm{oc}}=-\frac{1}{\ln n} \sum_{\mathrm{m}=1}^{\mathrm{n}} \mathrm{b}^{(\mathrm{m})}{ }_{\mathrm{ij}} \operatorname{lnyb}{ }^{(\mathrm{m})}{ }_{\mathrm{ij}} \\
\mathrm{w}_{\mathrm{oc}}=\frac{1-E_{\mathrm{oc}}}{\sum_{\mathrm{k}=1}^{\mathrm{n}}\left(1-E_{\mathrm{oc}}\right)}
\end{gathered}
$$

Get the objective weight of each index $\mathrm{w}_{\mathrm{o}}=\left(\mathrm{w}_{\mathrm{o} 1}, \mathrm{w}_{\mathrm{o} 2}, \cdots, \mathrm{w}_{\mathrm{om}}\right)$.

(3) combined weights

$$
\mathrm{w}_{\mathrm{c}}=\frac{\mathrm{w}_{\mathrm{sc}} \mathrm{W}_{\mathrm{oc}}}{\sum_{\mathrm{c}=1}^{\mathrm{m}} \mathrm{w}_{\mathrm{sc}} \mathrm{W}_{\mathrm{oc}}}
$$

Through the formula (9) to calculate the combination weight, and in the formula $\mathrm{W}_{\mathrm{c}}$ is satisfied that $0 \leq \mathrm{w}_{\mathrm{c}} \leq 1, \sum_{\mathrm{c}=1}^{\mathrm{m}} \mathrm{w}_{\mathrm{c}}=1$.

Step 4: weight the interval number, and find the interval number of the scheme to be compared.

The corresponding index interval number of the underlying index $\mathrm{c}_{\mathrm{j}}$ in the scheme $A_{\mathrm{i}}$ after normalized processing is recorded as $\mathrm{b}_{\mathrm{ij}}=\left(\mathrm{b}_{\mathrm{ij}}, \overline{\mathrm{b}_{\mathrm{ij}}}\right)$. The weight of the index is $\mathrm{w}$, and the interval number is weighted by the formula (10).

$$
c_{i j}=w_{i j} b_{i j}=\left(w_{i j} \underline{b_{i j}}, w_{i j} \overline{b_{i j}}\right)
$$

The interval number of the scheme $\mathrm{A}$ is calculated by the formula (11).

$$
\begin{aligned}
\mathrm{d}= & \mathrm{w}_{1}\left(\mathrm{c}_{11}+\mathrm{c}_{12}+\cdots+\mathrm{c}_{1 \mathrm{e}}\right) \\
& +\mathrm{w}_{2}\left(\mathrm{c}_{21}+\mathrm{c}_{22}+\cdots+\mathrm{c}_{2 \mathrm{~g}}\right)+\cdots \\
& +\mathrm{w}_{\mathrm{f}}\left(\mathrm{c}_{\mathrm{f} 1}+\mathrm{c}_{\mathrm{f} 2}+\cdots+\mathrm{c}_{\mathrm{fh}}\right)
\end{aligned}
$$

\section{Step 5: extract interval number information vector}

For interval numbers $\mathrm{d}=(\underline{\mathrm{d}}, \overline{\mathrm{d}})$, the interval number information vector $I_{\mathrm{d}}=\left(\mathrm{m}_{\mathrm{d}} \mathrm{Z}_{\mathrm{d}}\right)$ is extracted by the formula
(12) and (13).

$$
\begin{aligned}
& \mathrm{m}_{\mathrm{d}}=\frac{\underline{\mathrm{d}}+\overline{\mathrm{d}}}{2} \\
& \mathrm{z}_{\mathrm{d}}=\frac{\overline{\mathrm{d}}-\underline{\mathrm{d}}}{2}
\end{aligned}
$$

Step 6: determine the reference scheme and calculate the gray relational degree

Reference vector selection $\mathrm{m}_{\mathrm{cd}}=\max \left(\mathrm{m}_{\mathrm{d}}^{\mathrm{n}}\right), \mathrm{z}_{\mathrm{cd}}=\min \left(\mathrm{z}_{\mathrm{d}}^{\mathrm{n}}\right)$. Reference information vector is recorded as $I_{\mathrm{cd}}=\left(\mathrm{m}_{\mathrm{cd},}, \mathrm{z}_{\mathrm{cd}}\right)$.

The correlation degree between the program information vector and the reference information vector is calculated by using the formula (14) and (15) .

$$
\begin{aligned}
& \mathrm{d}\left(I_{\mathrm{d}}, I_{\mathrm{cd}}\right)=\sqrt{\sum_{\mathrm{i}=1}^{2}\left(I_{\mathrm{cd} i}-I_{\mathrm{d} i}\right)^{2}} \\
& \mathrm{r}\left(I_{\mathrm{d}}, I_{\mathrm{cd}}\right)=\frac{\alpha}{\alpha+\mathrm{d}\left(I_{\mathrm{d}}, I_{\mathrm{cd}}\right)}
\end{aligned}
$$

Where $\alpha$ is the resolution ratio, $\alpha>0$, in the paper $\alpha=0.1$. Step 7: rank relational degree sequence

The relational degree between the comparison scheme's information vector and the reference information vector should be calculated respectively. And quality of the scheme is represented by the gray relational degree. According to numerical value of the gray relational degree, correlation degree sequence should be ranked. Compared with the optimal reference information vector, the greater of the gray relational degree, the expressed scheme is better. Compared with the worst reference information vector, the smaller of the gray relational degree, the expressed scheme is better. And then the efficiency of the gray system is analyzed quantitatively.

Through above steps, the complex gray system can be decomposed into some indexes. Experts use the interval number to score for each index. Overcome the problem of the reliability of the score data are not enough because of the effect of the subjective and objective fuzzy factors in the traditional gray correlation theory by using a single numerical value. Using this method, the results of effectiveness evaluation quantitative analysis are more reasonable and reliable.

\section{Case Verification}

In this paper, the data comes from references [9-10] to analyze the results of the trajectory planning.From the results of the trajectory planning, the two groups of L1 and L2 which are of representative significance are selected. These two groups of trajectory planning are evaluated.

$\mathrm{L} 1$ represents the $3 \mathrm{UAVs}$ in the formation of the UAV doesn't simultaneously reach the goal for the collaborative planning of the track; L2 representing the UAVs arrives the goal at the same time. Assessment experts are five people, and the assessment schemes have two $n=2$. 
(1) Obtain expert evaluation matrix

Using the AHP method to establish an effective and reasonable hierarchical relationship, experts score for the 12 indexes of the index layer. Score control is over the range of $[0,10]$.Because the indexes are the benefit type index in this paper, the higher the value of the index is, the better the index will be. Thus, the expert evaluation matrix is obtained:

$$
\begin{aligned}
& X=\left[\begin{array}{llllll}
(6.7,7.0) & (4.7,5.0) & (7.27 .5) & (6.0,6.2) & (5.1,5.3) & (7.27 .5) \\
(6.5,6.7) & (4.8,5.2) & (7.27 .5) & (6.3,6.6) & (6.1,6.3) & (7.0,7.2)
\end{array}\right. \\
& \begin{array}{llllll}
(6.0,6.4) & (5.7,6.0) & (6.6,6.9) & (6.7,6.9) & (5.2,5.4) & (6.0,6.2)
\end{array} \\
& \begin{array}{llllll}
(6.5,6.7) & (5.8,6.2) & (6.8,7.2) & (6.7,6.9) & (5,3,5.6) & (6.2,6.5)
\end{array}
\end{aligned}
$$

The value of the interval number of each index is obtained by formula (1) and (2).

(2) Get the normalized score matrix of the expert by the formula (4).

(3) Determination of the weight of the index

$\mathrm{w} 1=(0.4899,0.2239,0.2862), \mathrm{w} 2=(0.7315,0.1809,0.0877)$,

$\mathrm{w} 3=(0.3602,0.6398), \mathrm{w} 4=(0.3266,0.1565,0.3671,0.1497)$,

$$
\mathrm{W}=(0.4153,0.3636,0.892,0.1318)
$$

(4) The interval numbers of the schemes to be compared are calculated by the formula (10)and(11).

$$
\begin{aligned}
& \mathrm{d}_{L 1}=(0.922406824486298,0.962074864244169) ; \\
& \mathrm{d}_{L 2}=(0.946046913847564,0.989928151057143)
\end{aligned}
$$

(5) In accordance with the formula (12), (13) to extract the information vector of the interval number of the scheme.

$$
\begin{aligned}
& I_{\mathrm{d}_{L 1}}=(0.942240844365233,0.019834019878936) ; \\
& I_{\mathrm{d}_{L 2}}=(0.967987532452353,0.021940618604790)
\end{aligned}
$$

(6) Determine the reference information vector $I_{\mathrm{cd}}=$ ( $0.967987532452353,0.019834019878936$ )

The gray relational degree is calculated by formula (14) and (15).

$$
\begin{gathered}
r_{L 1}=0.795249572940783 ; \\
r_{L 2}=0.979368632858783
\end{gathered}
$$

(7) Rank relational degree sequence which is concluded that the scheme L2 is closer to the ideal optimal scheme than the scheme L1 because $\mathrm{r}_{L 1}<r_{L 2}$. And the result is in line with the actual situation. The results of the difference methods are shown in table 1.

Table 1. Comparison of results obtained from methods.

\begin{tabular}{lll}
\hline & Traditional gray correlation degree & This paper \\
\hline L1 & 0.7913 & 0.7952 \\
L2 & 0.9149 & 0.9794 \\
\hline
\end{tabular}

\section{Conclusions}

In this paper, an improved effectiveness evaluation method based on the combination of AHP and grey interval correlation analysis is proposed. The evaluation index value is replaced a single value by judging interval, which is used to solve the problem of the reliability of the data that is caused by subjective and objective fuzziness in the traditional gray correlation theory. The concrete steps of effectiveness evaluation based on the improved method are given. Example comparison shows the effectiveness evaluation method based on the combination of AHP and grey interval correlation analysis is feasible. Because of the selection of the expert data that the interval array is used, the result is more reasonable and reliable.

\section{References}

[1] Zhang Jie, Tang Hong, Su Kai. Research on the method of effectiveness evaluation [M]. Beijing, National Defense Industry Press, 2009.

[2] Chen Qin, Zhao Yu-hui, Du Jun. "Application of the Improved Grey Correlation Analysis on Evaluating Radar Low Proability of Interception Performance "[J]. Fire Control Radar Technology, Vol. 41 No. 2 Jun, 2012. pp. $16 \sim 13$.

[3] Chang Shuang-Jun, Ma Jin-ya. Application Study on Synthetical Optimization Based on Grey Associated Method for Evaluating Effectiveness of Weapon system [J]. Journal of Sichuan Ordnance, Vol. 32 May, 2011. Pp.16 -19.

[4] Ning Xiao-lei, Wu Ying-xia, Chen Zhan-qi. Study on Validation of Simulation Models Based on Improved Grey Relational Analysis [J]. Computer Simulation, Vol.32.Jul, 2015 pp. $259-263$.

[5] ChihHsuan Wang. A systematic approach to select the optimal project portfolios for green manufacturing: An empirical study on TFT-LCD fabrication processes. Proceedings of the 2015 International Conference on Industrial Engineering and Operations Management Dubai, UAE, March 3 - 5, 2015.

[6] Wu Jiang, Huang Deng-shi. Review of the Research on the Method of Interval Number Ranking [J]. Systems Engineering, Vol. 22, 2004, pp.1-4.

[7] Xu Rui-li, Xu Ze-shui. Study on the practice and recognition of Mathematics in Practice and Theory [J], Vol.37 No.24 Dec, 2007. pp. $1 \sim 7$.

[8] Chen Chun-fang, Zhu Chuan-xi. Method of Interval Number Ranking Based on Vector Similarity and Application [J]. Statistics and Decision, 2014 (3). pp.76-78.

[9] Wu Jing. Research on Trajectory Planning and Effectiveness Evaluation for Multi-UAV Cooperative [D]. Nanchang: Nanchang Hangkong University, June, 2012.

[10] Huang Guo-qing, Su Lin. "Analysis of Effectiveness Evaluation Based on Improved Gray Synthesize Relational Degree [J]". Command Control \& Simulation, 2015(3). pp 90-93. 